\title{
Focus sur six « reflets » de la vie de la SFP au printemps 2014
}

Au cœur de la vie de la SFP, se trouve, essentielle, l'action de chacun des physiciens se mobilisant pour renouveler et poursuivre les actions de notre société. L'investissement, bénévole, des femmes et des hommes, est vital pour que vivent les sections locales, les divisions et les commissions. C'est donc avec plaisir et beaucoup de remerciements que, le 20 mars 2014, la SFP a accueilli au sein de son conseil d'administration : Pierre-François Cohadon, Stéphanie Couvreur, Marie-Renée de Backer, Pascal Gay, Dave Lollman, Henri Mariette, Patrick Rairoux et Jean-Paul Duraud, également membre du bureau.

La Société Française d'Optique (SFO) et la Société Française de Physique (SFP) se sont coordonnées pour que leurs congrès généraux biannuels alternent dès 2016. En 2015, les semaines retenues sont différentes, en juillet pour la SFO, en août pour le Congrès général de la SFP, qui aura lieu à Strasbourg, du 24 au 28 août 2015. Le Conseil scientifique, émanation des divisions de la SFP, présidé par Daniel Estève, s'est mis au travail dès le 9 avril 2014 et se réunira de nouveau fin juin 2014.

Saluons le travail énergique d'Annick Suzor-Weiner, grâce à qui l'accord de la mi-avril 2014 entre l'American Physical Society et la SFP a pu être signé : cet accord donne aux adhérents de chaque société les mêmes " privilèges » lors de nos colloques réciproques, soumissions de publications et autres évènements pilotés par chacune des sociétés savantes. La SFP a immédiatement diffusé par courrier électronique cette annonce importante à plus de 200 directeurs de laboratoires.

Les $14^{\text {es }}$ Journées SFP-Matière Condensée, élargies à la dimension européenne (JMC14-CMD25, Paris, 20-24 août 2014) accueilleront au sein d'une table ronde dédiée au "Future of Physics Publishing", le Pr Gene Sprouse, éditeur en chef des journaux de l'APS, Eberhardt Bodenschatz, éditeur en chef du New Journal of Physics, et des représentants de I'European Physical Society et de la SFP.

À la fin de l'été 2014, le nouveau site web de la SFP sera opérationnel. Début avril 2014, la SFP a recruté Mayline Gautié, titulaire d'un master en management, spécialité Communication, pour assurer la vie de ce nouveau site, qui sera régulièrement enrichi par les annonces des colloques et évènements, mais aussi grâce aux apports de nos divisions, commissions et sections locales.

Le 22 avril 2014, à l'École de Chimie de Paris (Chimie Paris-Tech) s'est déroulée une journée consacrée aux quatre générations scientifiques Friedel : Charles, Georges, Edmond et Jacques, dans le contexte de l'Année Internationale de la Cristallographie, en présence d'Alain Fuchs, Président du CNRS et d'Yves Bréchet, HautCommissaire à l'énergie atomique. Toutes les communautés de physiciens, de chimistes et des autres disciplines sont totalement conscientes de l'apport essentiel des Friedel au développement de la science française pour l'amener au meilleur niveau international. L'intervention des orateurs fut chargée d'émotion et a illustré, trop sommairement sans doute, l'ensemble des disciplines enrichies par les travaux et découvertes des membres de la famille Friedel, honorés ce jour. La SFP s'est associée avec ferveur à cet évènement exceptionnel. Tous les participants ont apprécié la présence chaleureuse de Jean et Paul Friedel, les fils de Jacques, de leurs enfants et petits-enfants, qui ont contribué à pallier l'absence de Jacques Friedel auquel nous souhaitons un prompt rétablissement. Un grand merci aux organisateurs, en particulier à Olivier Hardouin Duparc et Valérie Cabuil. C'est également un plaisir de rappeler les récentes cérémonies de remises de prix qui honorent les lauréats et contribuent très largement au rayonnement de la SFP. Le Prix Gentner Kastler a été remis le 19 mars à Berlin, à François Biraben pour ses innovations au sein de la spectroscopie de précision des transitions atomiques et dans les mesures des constantes fondamentales. Le prix Jean Perrin a été décerné le 7 avril à Lille, à Daniel Hennequin pour ses remarquables actions pour la diffusion de la culture scientifique. Le prix Félix Robin 2012, le 29 avril à Paris, est revenu à Jean-Pierre Lasota, pour ses travaux très originaux dans le domaine des naines blanches, étoiles à neutrons et trous noirs.

Alain Fontaine

Président de la Société Française de Physique (SFP) 